\title{
Weibo: La herramienta del cambio
}

\author{
Patricia Castro Obando \\ (Corresponsal del diario El Comercio en China)
}

Recibido: 30/1/2012

Aprobado: 27/2/2012

\begin{abstract}
Resumen: Este artículo se concentra en la plataforma digital por excelencia, que está cambiando la dinámica de la distribución de la información en China: Weibo. Se trata de un servicio de microblogging local, con características similares al Twitter pero adaptado al consumidor chino y a las regulaciones gubernamentales. En un país que controla los medios de comunicación, Weibo genera un nuevo formato para difundir noticias, ponerlas en la agenda nacional y bloquear hasta la propia censura. La herramienta está en manos de 513 millones de internautas chinos.
\end{abstract}

Palabras clave: Microblogging / Weibo / China / censura

\section{Weibo: The tool for change}

Summary: This article focuses on the main digital platform that is changing the dynamics of the distribution of information in China: Weibo. This is a local microblogging service, with similar characteristics to Twitter but adapted to the Chinese consumer and government regulations. In a country that controls the media, Weibo generates a new format for broadcasting news, put them on the national agenda and even block the censorship. The tool is in the hands of 513 million Chinese internet users.

Keywords: Microblogging / Weibo / China / censorship 


\section{Introducción}

$\mathrm{N}$ ada genera mayor dolor de cabeza a los censores de internet en China que el servicio de microblogging Weibo. El aparato gubernamental que controla la información calificada como sensible está perdiendo su eficacia frente a esta herramienta que logra difundir velozmente las noticias censuradas por los medios estatales. En los últimos meses, el Gobierno ha reforzado su control sobre los microblogs pero con 513 millones de internautas, de los cuales más de la mitad utiliza el teléfono celular para conectarse a la red, la batalla recién empieza. Este artículo, que actualiza los datos de mi tesis de maestría "La internet en China" (Castro Obando 2011a, 2011b), presenta tres secciones para explicar la importancia del Weibo: la red china, Weibo vs. Twitter y el caso "Mi padre es $\mathrm{Li}$ Gang".

\section{La red china}

En China la internet está compuesta por 513 millones de usuarios (hasta diciembre de 2011), de acuerdo con los datos proporcionados por el Centro de Información de la Red de Internet de China (CNNIC, por sus siglas en inglés), una agencia gubernamental creada en 1997 que pertenece al Ministerio de la Industria de la Información.
Esta tendencia ascendente genera un proceso muy intenso y sui géneris de evolución y cambio. El último reporte elaborado por el CNNIC y publicado en su página web (CNNIC 2011, en línea) arroja los siguientes datos sobre el uso de internet en China hasta diciembre del 2011:

- 513 millones de internautas chinos.

- 356 millones de internautas se conectan por teléfonos celulares.

- 392 millones de internautas que se conectan a través de la computadora.

- 136 millones de internautas rurales.

- 775 millones de nombres de dominio en China.

- 230 millones de sitios web en territorio chino.

- 18,7 horas de media semanal permanece conectado un internauta chino.

Los gráficos que aparecen en las páginas siguientes han sido tomados del reporte de enero del 2012 del CNNIC y ayudan a ilustrar mejor lo dicho.

En China existen 513 millones de usuarios. Las columnas en azul muestran el número de internautas y las líneas rojas el porcentaje de penetración. Se calcula que China tiene una población de 1.400 millones de habitantes, por lo tanto, según estas últimas estadísticas, casi el cuarenta por ciento de la población está conectada a internet. 


\section{Gráfico 1}

Número de internautas chinos

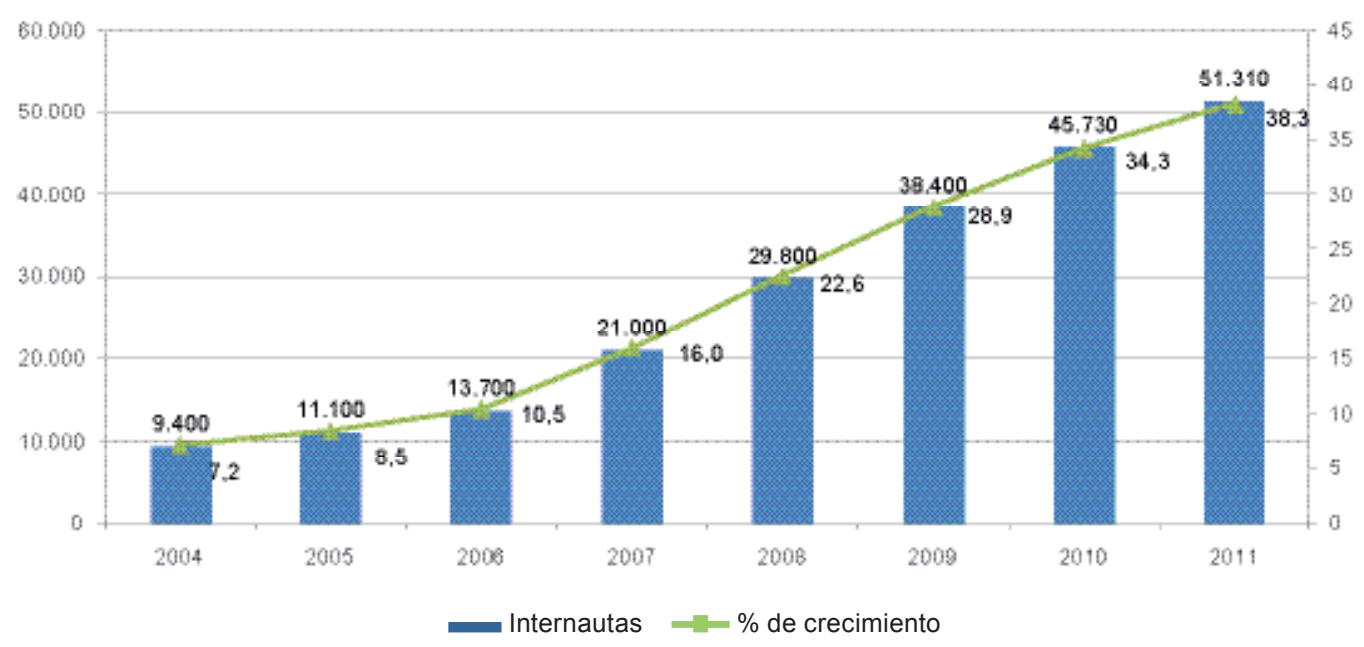

Por otro lado, el uso de teléfonos ro de internautas que utilizan un tecelulares en China está bastante geléfono celular; y la línea roja expresa neralizado; a la fecha existen casi 356 el índice de crecimiento. Es notable la millones de usuarios. Obsérvese en el gráfico estas contrastantes cifras: las disminución del crecimiento en tan solo cuatro años. columnas color azul indican el núme-

\section{Gráfico 2}

Internautas que utilizan teléfonos celulares

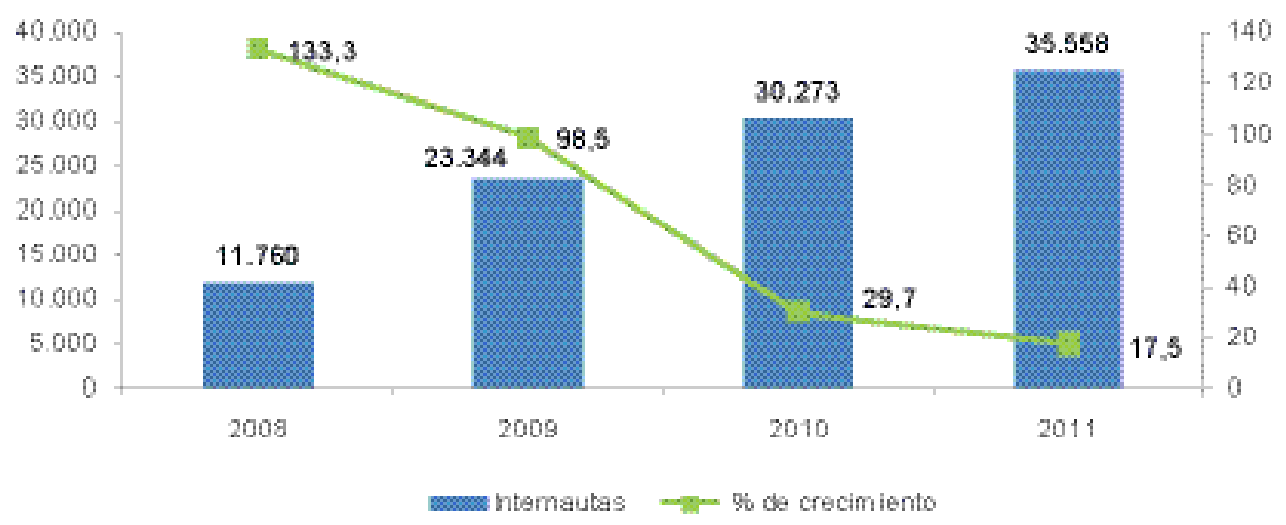




\section{Gráfico 3 \\ Edad del internauta \\ (en porcentajes)}

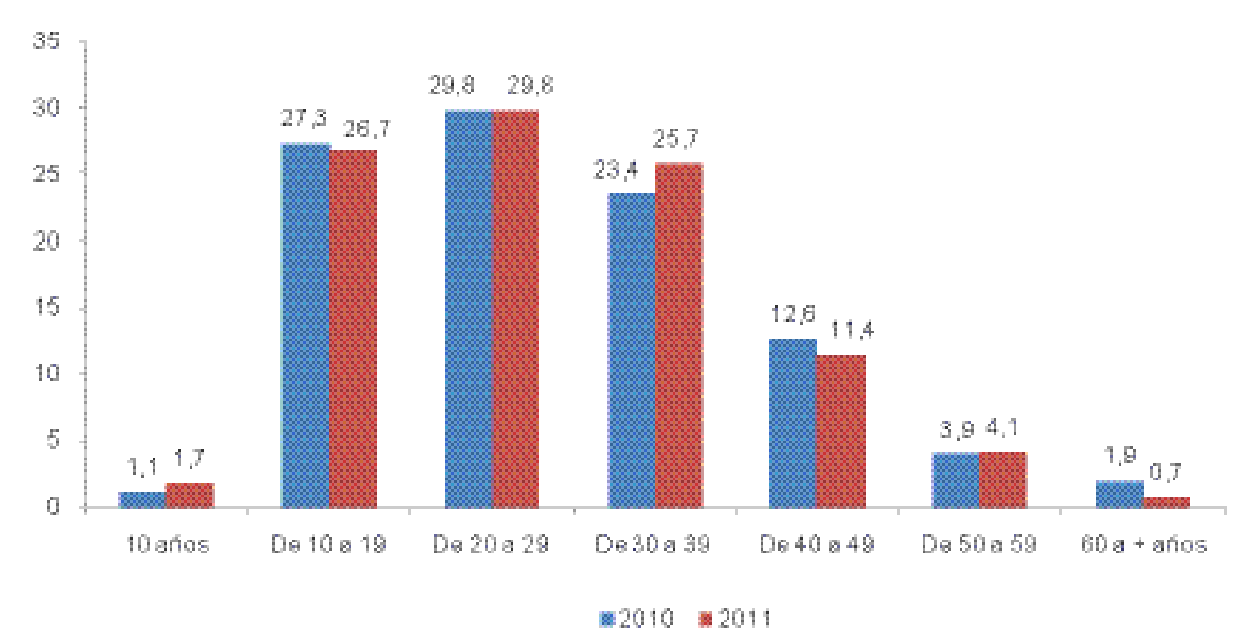

En cuanto a las edades de los inter- entre 10 y 39 años (población joven en nautas, nótese que el segmento de 30 a 39 años de edad ha aumentado más de dos puntos porcentuales en tan solo un año. Pero el segmento que sigue liderando el uso de internet es el de 20 a 29 años de edad. En síntesis, cuatro de cada cinco internautas chinos tienen su mayoría).

En el último año, los usuarios varones han aumentado 0,1 por ciento, mientras que las mujeres han disminuido en la misma proporción. Un poco más de 11 puntos porcentuales separan a ambos grupos.

Gráfico 4

Hombres vs. mujeres

(en porcentajes)

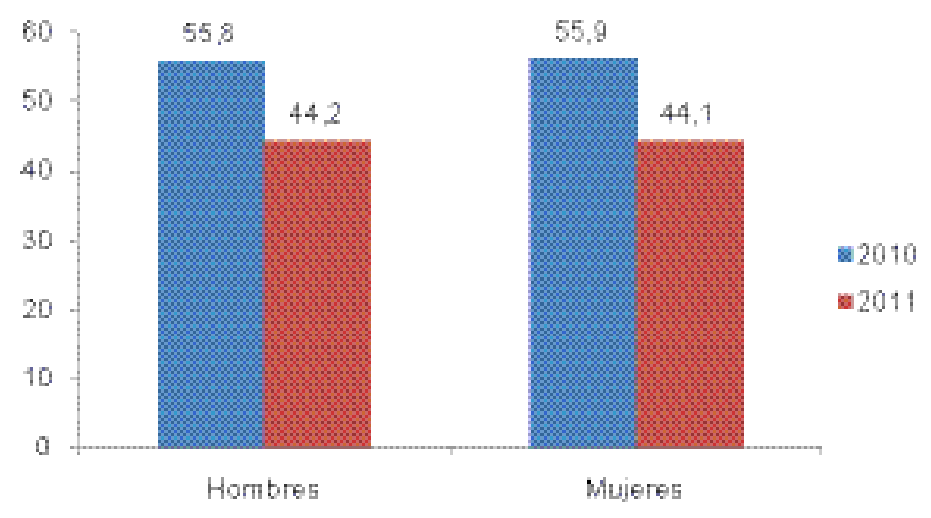




\section{Gráfico 5 \\ Ocupación del internauta \\ (en porcentajes)}

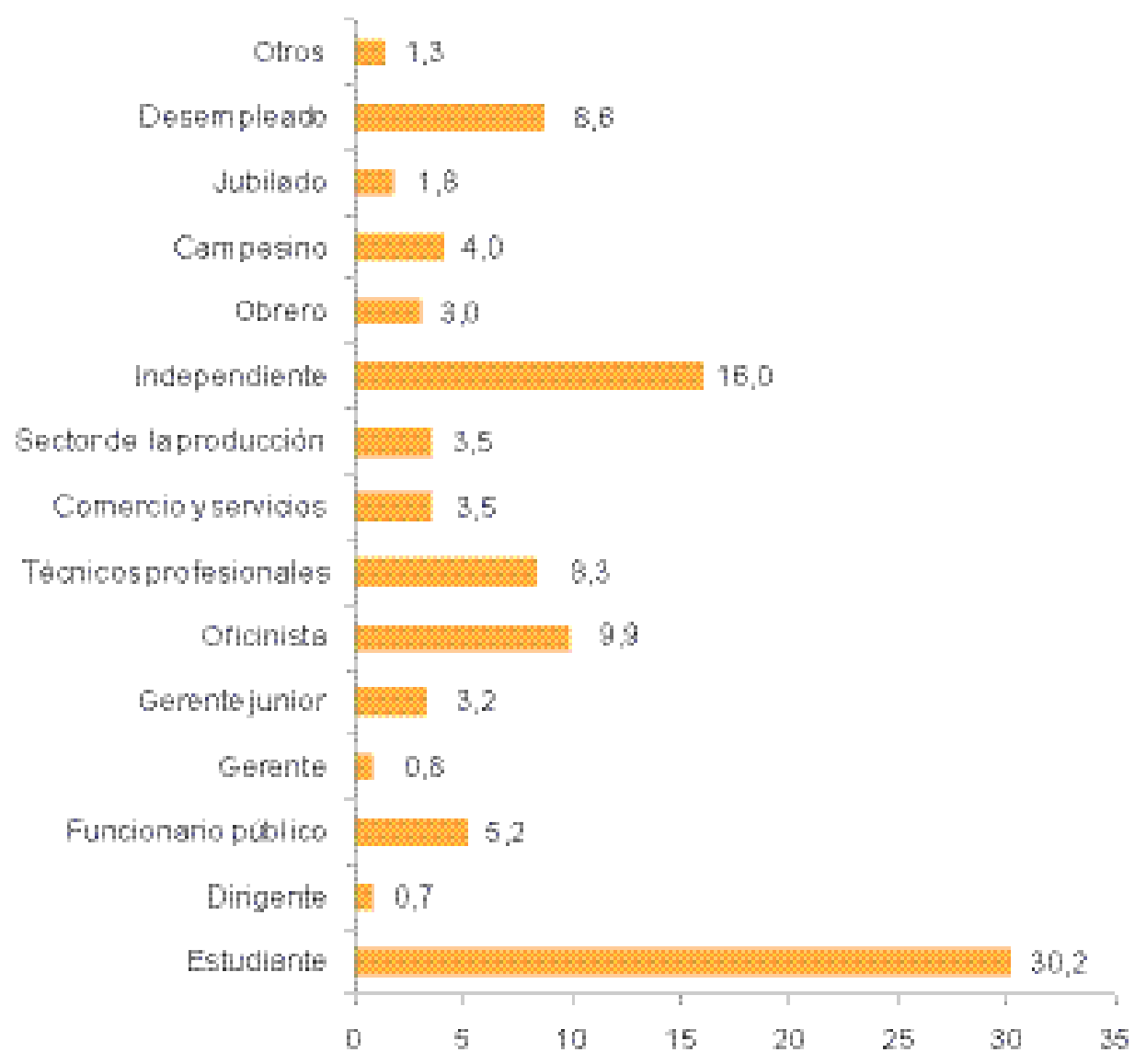

Según se observa en el gráfico 5 trabajadores independientes, con 16,0 referido a la ocupación del usuario, el por ciento. Y solo el 0,7 por ciento son 30,2 por ciento de los internautas chi- usuarios que trabajan para organizanos son estudiantes. El segundo gru- ciones gubernamentales en puestos po más numeroso lo conforman los dirigenciales. 


\section{Cuadro 1}

Principales usos dados a la red por los internautas chinos

\begin{tabular}{|c|c|c|c|c|c|}
\hline \multirow[b]{2}{*}{ Actividades } & \multicolumn{2}{|c|}{2011} & \multicolumn{2}{|c|}{2010} & \multirow[b]{2}{*}{$\begin{array}{l}\text { Crecim. } \\
\text { anual } \%\end{array}$} \\
\hline & $\begin{array}{c}\text { Usuario } \\
\text { (en millones) }\end{array}$ & $\%$ & $\begin{array}{c}\text { Usuario } \\
\text { (en millones) }\end{array}$ & $\%$ & \\
\hline Buscador & 41510 & 80,9 & 35258 & 77,1 & 17,7 \\
\hline Mensajería instantánea & 40740 & 79,4 & 37453 & 81,9 & 8,8 \\
\hline Música & 38585 & 75,2 & 36218 & 79,2 & 6,5 \\
\hline Noticias & 36687 & 71,5 & 35304 & 77,2 & 3,9 \\
\hline Juegos & 32531 & 63,4 & 28398 & 62,1 & 14,6 \\
\hline Videos & 32428 & 63,2 & 30410 & 66,5 & 6,6 \\
\hline Blog & 31864 & 62,1 & 29450 & 64,4 & 8,2 \\
\hline Microblog & 24988 & 48,7 & 6311 & 13,8 & 296 \\
\hline Correo electrónico & 24577 & 47,9 & 24969 & 54,6 & $-1,6$ \\
\hline Redes sociales & 24424 & 47,6 & 23505 & 51,4 & 3,9 \\
\hline Literatura & 20267 & 39,5 & 19481 & 42,6 & 4,0 \\
\hline Compras & 19395 & 37,8 & 16051 & 35,1 & 20,8 \\
\hline Pagos & 16676 & 32,5 & 13719 & 30 & 21,6 \\
\hline Banca en línea & 16624 & 32,4 & 13948 & 30,5 & 19,2 \\
\hline Foros & 14469 & 28,2 & 14817 & 32,4 & $-2,3$ \\
\hline Compras en grupo & 6465 & 12,6 & 1875 & 4,1 & 244,8 \\
\hline Reserva de viajes & 4207 & 8,2 & 3613 & 7,9 & 16,5 \\
\hline Acciones & 4002 & 7,8 & 7088 & 15,5 & $-43,5$ \\
\hline
\end{tabular}

El internauta chino usa la red principalmente para buscar información, enviar mensajes instantáneos, escuchar música y leer noticias. Sin embargo, en el último año se puede notar un impresionante crecimiento de 296 por ciento en el servicio de microblog- ging. Es decir, hay casi 250 millones de usuarios que utilizan Weibo (cerca de la mitad de los internautas chinos), y también es interesante el crecimiento de 244,8 por ciento en el rubro de compras en grupo, donde ya existen 65 millones de usuarios. 


\section{Gráfico 6 \\ Actividades a través del teléfono celular \\ (en porcentajes)}

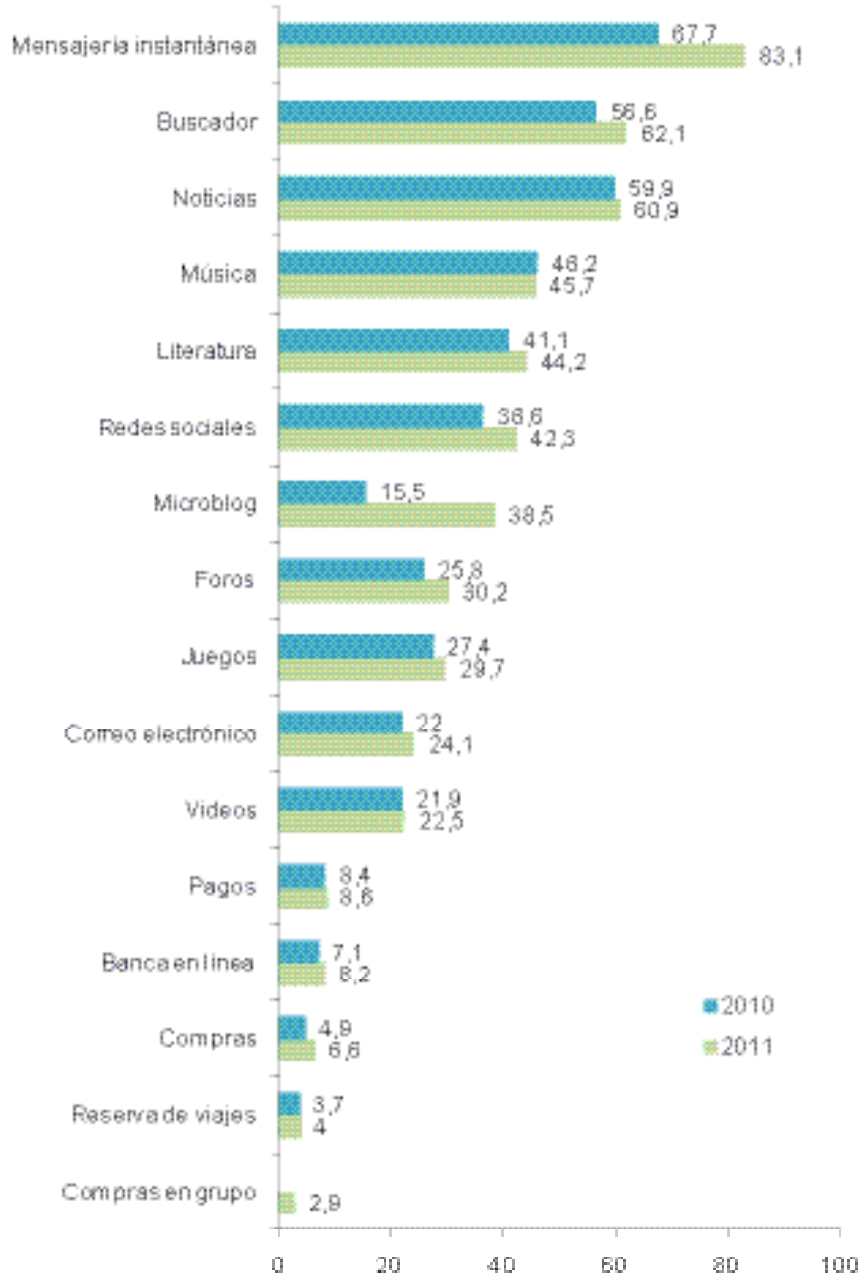

De acuerdo con este gráfico refela lectura de noticias en línea, con 60,9 rente al uso de la telefonía celular en China, se puede observar que la actividad que lidera el panorama de telefonía inalámbrica es la mensajería instantánea, con 83,1 por ciento de uso. Le siguen la utilización del teléfono como buscador, con 62,1 por ciento, $y$ por ciento. Si bien el microblog Weibo aparece en séptima posición, hay que observar que solo en un año ha crecido de 15,5 por ciento a 38,5 por ciento, convirtiéndose en la actividad que más se ha desarrollado a través de los teléfonos celulares. 


\section{Weibo vs. Twitter}

Weibo, que se traduce como "microblog", es el servicio de microblogging en la internet china. Si bien existe una variedad de "weibos": Tencent Weibo, Sohu Weibo, entre otros, actualmente el más popular es Sina Weibo, que en chino se conoce como Xinlang Weibo.

Perteneciente a la Corporación Sina, Sina Weibo fue lanzado en agosto del 2009 con características de Twitter y de Facebook. Esta nueva criatura, que fusionaba dos clones en uno, ha conseguido en apenas un par de años unos 250 millones de usuarios registrados (Bishop 2011).

Debido a su popularidad, Sina Weibo presentó en abril del 2011 su propio dominio weibo.com, que reemplaza al anterior que contenía su nombre. Ya no lo necesita, ahora es el Weibo por excelencia.

La Corporación Sina aprovechó la ausencia definitiva de Twitter y el bloqueo temporal de Fanfou, el primer Weibo que apareció en la red china, para lanzar una herramienta que concentraba a los dos más populares servicios digitales a escala mundial (Twitter y Facebook) en una sola plataforma.

En corto tiempo Sina Weibo dio el salto de la etapa de clonación a otra de adecuación. Además de los 140 caracteres y de la lista de seguidores, clásicos de Twitter, esta plataforma china le ha sumado otras características.

\section{El microblog Weibo}

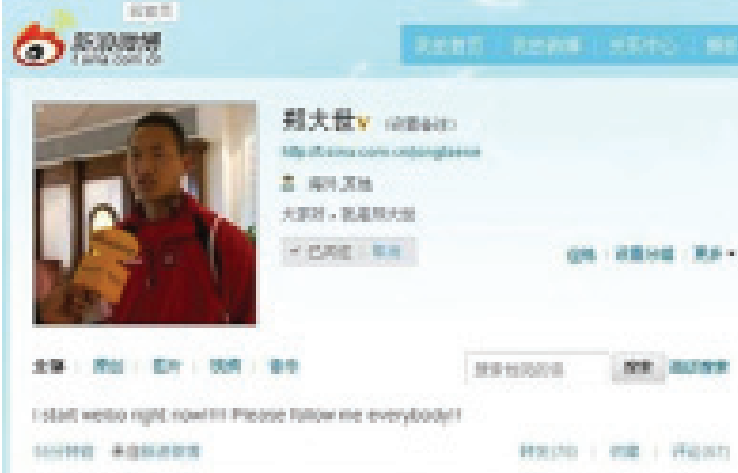

Fuente: <http://sports.sina.com.cn/n/2011-01-12/02175 406158.shtml>.

Los usuarios pueden insertar gráficos con sus emociones o adjuntar archivos de música, videos y fotos en cada post para compartirlos con sus seguidores sin necesidad de una aplicación externa.

Sina Weibo ha logrado construir una plataforma que ordena, clasifica y segmenta la información para el usuario, mucho más que Twitter o Facebook. Por ejemplo, los comentarios a cada post de este Weibo aparecen colgados como una lista, al estilo de las redes sociales. Esto permite rastrear con mayor facilidad el post original y saber su movimiento en la red.

Un movimiento clave de Sina Weibo que le dio ventaja sobre sus competidores fue convencer a las estrellas chinas del mundo real (China continental, Hong Kong, Macao y Taiwán) para que abrieran cuentas personales en esta plataforma. 
De acuerdo con sus propias estadísticas, que aparecen en su página web, más de 5.000 compañías y 2.700 organizaciones de medios de comunicación en China tienen una cuenta activa en Weibo (Wang 2010).

En marzo del 2011, Sina Weibo superó los 100 millones de usuarios. Ese mismo mes acaparó el 56\% del mercado de microblogs en la internet china. En junio del 2011, la plataforma anunció que lanzará un servicio en inglés (Wang 2010).

Como ha sucedido con otras herramientas locales, Sina Weibo también ha ampliado sus servicios. La plataforma ofrece "Weilingdi", una aplicación de localización similar a Foursquare, y "Sina Weibo Dama”, exclusivamente para las mujeres.

Es la herramienta que mejor ha manejado el tema de la censura. No ha prescindido de ella porque no puede, aunque tampoco la aplica con el rigor de RenRen, QQ o Baidu. Esto se debe a la necesidad que tiene de generar polémica en la red.

\section{El caso "Mi padre es Li Gang"}

El caso "Mi padre es Li Gang" (Castro Obando 2010) muestra el poder que han adquirido en China los medios sociales digitales descritos anteriormente, liderados por el Weibo. Su triunfo, particularmente en este país, radica en haber asumido el papel de vehículo de denuncia que corresponde a los tradi- cionales medios de comunicación, los que se encuentran sujetos a un mayor control gubernamental.

Propagada inicialmente a través del microblogging, superando la muralla de la censura, esta historia puso sobre el tapete un episodio de injusticia social. Los internautas locales que comandaron esta campaña utilizaron la internet china como soporte de cohesión $\mathrm{y}$, a la vez, válvula de escape del descontento social.

Weibo se coronó como una efectiva plataforma de intercambio de información y denuncia pese a que también se encuentra sometida a las regulaciones del país. Los medios sociales chinos han impulsado un mecanismo que cada vez más está escapando al control de los reguladores chinos.

La historia de "Mi padre es $\mathrm{Li}$ Gang" puede resumirse de la siguiente forma: un joven de la clase media atropella a dos muchachas de origen humilde dentro de un campus universitario, intenta huir y cuando es detenido por otros estudiantes, reta a los testigos diciendo: "Mi padre es $\mathrm{Li}$ Gang".

La secuencia del hecho se desarrolló más o menos de la siguiente forma:

- Suceso.- El joven que cometió el atropello, de 22 años de edad, es el hijo de Li Gang, el subdirector de Seguridad Pública de la ciudad de Baoding. Tras haber consumido licor, conducía su auto a gran velocidad dentro del campus de la 
Universidad de Hebei y fue en esas condiciones que atropelló a dos estudiantes, pero no se detuvo a auxiliarlas. Cuando intentaba abandonar el campus fue interceptado por alumnos y guardias de seguridad, quienes bloquearon la puerta y lo obligaron a bajar del coche. Fue entonces que el joven amenazó a los testigos con su famosa frase "Mi padre es Li Gang".

- Denuncia.- Nadie se atrevió a acudir a la policía o alertar a los medios pero los testigos denunciaron el episodio en Weibo, redes sociales, foros de internet, mensajería instantánea y otras plataformas. Cada hora, millones de internautas se sumaban a la cadena para replicar la información, en lo que se conoce como "efecto racimo".

- Polémica.- Los internautas propagaron la frase "Mi padre es Li Gang" a través de las distintas plataformas digitales para mostrar los profundos desequilibrios que sufre la sociedad china. La víctima era hija de un campesino y el victimario es hijo de un funcionario del gobierno, lo cual hasta cierto punto lo protegía ante los medios tradicionales y la policía.

- Reacción.- El caso nunca hubiese trascendido de no ser por la red. Los internautas asumieron la labor de periodistas, policías y hasta jueces en internet pero con resultados concretos en el mundo real. Tal fue el malestar de la opinión pública ante esta injusticia que las autoridades finalmente optaron por atender el expediente "Mi padre es $\mathrm{Li}$ Gang". A partir de entonces la policía actuó con mayor determinación y el caso recibió cobertura en los medios tradicionales. El Gobierno prometió un juicio justo.

Estos cuatro grandes momentos: suceso, denuncia, polémica y reacción, pueden ser subdivididos en instancias más precisas o alternar -con excepción de los extremos- ligeramente de posición. Lo destacado es que este motor se activa precisamente en internet a través de herramientas como Weibo y otras.

Las nuevas plataformas permiten "democratizar la información" en una sociedad acostumbrada a un diálogo en una sola dirección:

Gobierno

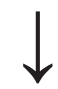

Población

\section{Conclusiones}

El expediente "Mi padre es Li Gang" es una prueba del poder de la internet en China y la efectividad de los medios sociales chinos como canales de intercambio de información, ante el criticado rol que ejercen los medios 
tradicionales de comunicación sujetos al control gubernamental.

En este caso, y en muchos otros, se ha seguido un patrón que cada vez cobra más fuerza en el país:

- Sucede un hecho polémico.

- Los medios tradicionales no informan.

- Uno o más internautas, utilizando los medios sociales locales, hace la denuncia en la internet china.

- Otros internautas replican la misma noticia en diversos soportes.

- La censura actúa, pero debido al "efecto racimo" (reproducción instantánea y veloz) el caso ya ha traspasado las fronteras digitales y se conoce en el mundo real.

- Se genera polémica y la sociedad exige justicia.

- Las autoridades ordenan a la policía atender el expediente.

- Los medios tradicionales cubren la noticia.

- El caso, por lo general, recibe justicia.

Lo que sugiere este patrón anterior es que las herramientas chinas son parte de un motor de cambio, como ya ha sucedido en otras sociedades. Ahora todo dependerá del internauta chino, que también se encuentra inmerso en una evolución y ha emprendido su propio camino, el cual tarde o temprano lo llevará más allá de las fronteras chinas.

\section{Bibliografía}

Bishop, Bill (2011). “Inside Sina Weibo [diapositivas]". <http://digicha.com/ index.php/2011/02/inside-sina-weibo/s. [Consulta: 30 de noviembre del 2011.]

Castro Obando, Patricia (2011). “La internet en China: ¿El reflejo del espejo? Una aproximación comparativa de las plataformas digitales chinas y sus pares extranjeras". Tesis para optar el grado de magíster en Comunicaciones. Lima: Pontificia Universidad Católica del Perú.

—. (10 de noviembre del 2011). “EEl reflejo del espejo?". Prezi. <http:// prezi.com/1gknjdkif6ns/el-reflejodel-espejo/>. [Consulta: 30 de enero del 2012].

-. (7 de noviembre del 2010). "Mi padre es Li Gang". El Comercio. Lima, p. B6.

Centro de Información de la red de Internet de China. "Informe 29". <http://www.cnnic.cn/dtygg/dtgg/ 201201/W020120116337628870651. pdf $>$.[Consulta: 30 de enero del 2012].

WANG, Shanshan (7 de febrero del 2010). "Sina's microblogging power". Caixin online. <http://english.caixin. cn/2010-07-02/100157821.html>. [Consulta: 30 de noviembre del 2011]. 